\title{
STUDYING PULSAR EMISSION REGIONS USING INTERSTELLAR SCATTERING
}

\author{
CARL R. GWINN \\ University of California, Santa Barbara
}

\begin{abstract}
Radio-wave scattering in the interstellar plasma provides the means to circumvent the diffraction limit for earth-based instruments, and to image the emission regions of pulsars. For the past 25 years, observers have sought to exploit this fact to learn how pulsars shine. I review the techniques developed, and summarize measurements of size of emission regions of pulsars to date.
\end{abstract}

\section{Introduction}

By unknown processes, pulsars convert a tiny fraction of their rotational energy into radio and shorter-wavelength emission. The density fluctuations of the interstellar refract radio waves, and so act as a huge but corrupt lens. This lens offers nanoarcsecond resolution at the pulsar. Observers have long attempted to exploit this potential to provide observational constraints on the emission process. They have developed several techniques, and found a variety of interesting results, as summarized below.

Many astrophysical researchers play roles analogous to herbivores, consuming vast amounts of nearly uniform data. Some are more like carnivores, rarely digesting small amounts of richer data. In this review I seek to broaden the paradigm to include creatures more like baleen whales, which consume large amounts of relatively nourishing, but perhaps not completely uniform, data.

\section{Radius-to-Frequency Mapping}

Observations of pulse profiles and their variations with time, frequency, and Stokes parameters provide many useful, model-dependent, constraints on pulsar emission mechanisms. Among these, I will describe the radius-to-frequency mapping. Measurements based on this scenario provide an interesting contrast to results from scintillation studies.

Theory suggests that pulsar emission arises from relativistically-outflowing electron-positron plasma on the 'open' magnetic field lines. These lines link a pulsar's surface to the cylinder corotating at light speed. In the cone of open field lines, lower radio frequencies may be generated at lower plasma densities. For example, 
the emission frequency at a given point may be some multiple of the plasma frequency there. The magnetic field lines diverge, so at lower frequencies the emission region may be at higher altitudes (and larger in size). If the emission region is higher, and so closer to the observer, the lower frequencies should arrive at the observer earlier - even after removing the best-fit values for the time delay due to interstellar dispersion. A variety of other effects, including variations in intrinsic pulse shape, aberration, relativistic beaming, lateral bending of the cone of open field lines, and the fraction of the cone that the emission region occupies, also can affect the observed arrival time as a function of frequency. Phillips (1992) discusses these effects.

From measurements of pulse arrival times at observing frequencies of $f=47$ to $4800 \mathrm{MHz}$, Phillips concluded that emission zones over this frequency range are separated by no more than $200 \mathrm{~km}$ in pulsars B0823+26, B0919+06, B0950+08, and $\mathrm{B} 1133+16$. Under the assumption of a dipolar magnetic field, with emission filling the open field lines, and emission frequency $f \propto n^{\xi}$, where $n$ is plasma density and the power-law index $\xi \leq 0.66$, he finds that the emission altitude must be less than $100 \mathrm{~km}$ in these objects.

\section{Source Structure from Scattering}

Radio-wave scattering in the interstellar plasma produces a diffraction pattern in the plane of the observer. Observers have used 3 measures of this diffraction pattern to probe pulsar size:

- shift of fringes over the pulse, during dringing events,

- shift of the usual random pattern over the pulse, and

- smearing of the pattern, and consequent decrease in modulation, by source size.

The first 2 techniques measure the shift of the centroid of the emission region over the pulse. The third measures the size of the emission region. Figure 1 illustrates these techniques. I discuss each below, with examples.

\subsection{FRINGING EPISODES}

Occasionally two paths through the interstellar medium dominate radiation received at the observer. These paths interfere to produce fringes in the observer plane. If the emission region shifts during the course of a pulse, perhaps as the pulsar rotates the emission region across the line of sight, one path shortens and the other lengthens, shifting the fringes in the observer plane.

Wolszczan and Cordes (1992) observed an episode of fringing in pulsar B 1237+ 25. They observed a shift of the fringe pattern over the pulsar pulse, on two days of observations. On each day, the change in fringe phase was proportional to the reciprocal of the fringe spacing, as expected for the same shift at the source and 


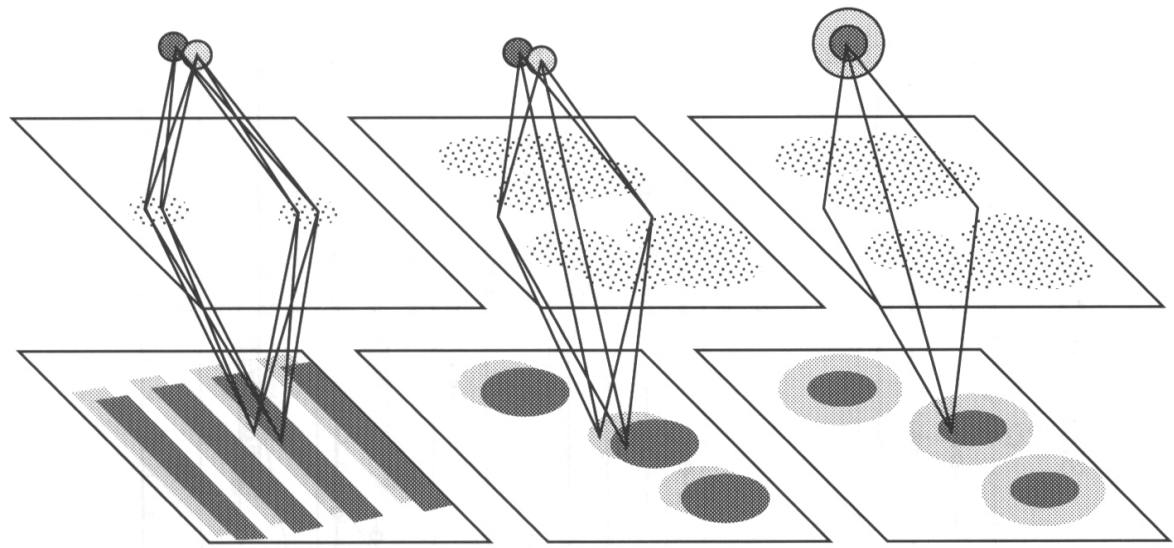

Figure l. Left: Two ray paths through the interstellar medium produce interference fringes in the plane of the observer. Source motion produces a reflex shift of the fringes. Middle: Source motion produces a reflex shift of the random interference pattern. Right: Source size smears the pattern, as compared to that from a point source.

different separations of paths, on the two days. The change in fringe phase did not vary monotonically over the pulse, and so was inconsistent with emission along dipole magnetic field lines at the pulsar. They found a shift of less than $1100 \mathrm{~km}$ at $f=430 \mathrm{MHz}$. The unknown location of the refractor along the line of sight is responsible for most of the uncertainty of the result. The fact that the emission region must lie within the light cylinder provides an important constraint on that location.

Gupta et al. (1999) report a fringing event on pulsar B1133+16. They also observe a shift of the fringes over the pulsar pulse. From the slopes of the scintillations in the frequency-time diagram, they derive the bending angles of the interfering rays, and so the distance of the refractor. They find that the refractor lies at $77 \%$ of the distance of the pulsar, near the edge of the Local Bubble. They also observed a non-monotonic shift of the fringes, again ruling against dipole structure for the pulsar's magnetic field. They determined a source size of about $300 \mathrm{~km}$ at $f=330 \mathrm{MHz}$. Wolszczan et al. (1988) also observed B1133+16 during a fringing event. They detected a shift of the fringes over the pulse, and inferred a size of $<3 \times 10^{4} \mathrm{~km}$ at $f=430 \mathrm{MHz}$.

\subsection{MOTION OF RANDOM SCINTILLATION PATTERNS}

Usually the diffraction pattern is random. Motion of the source produces a reflex shift of the pattern. Comparison of the scintillation pattern at 2 or more pulse phases can detect a synchronous shift of the emission region.

Cordes et al. (1983) compared diffraction patterns in 2 gates on the sub-pulses of pulsars B $0525+21$ and B1133+16, at $f=430 \mathrm{MHz}$. They compared autocorrelations of the scintillations in each gate with cross-correlation between gates. 


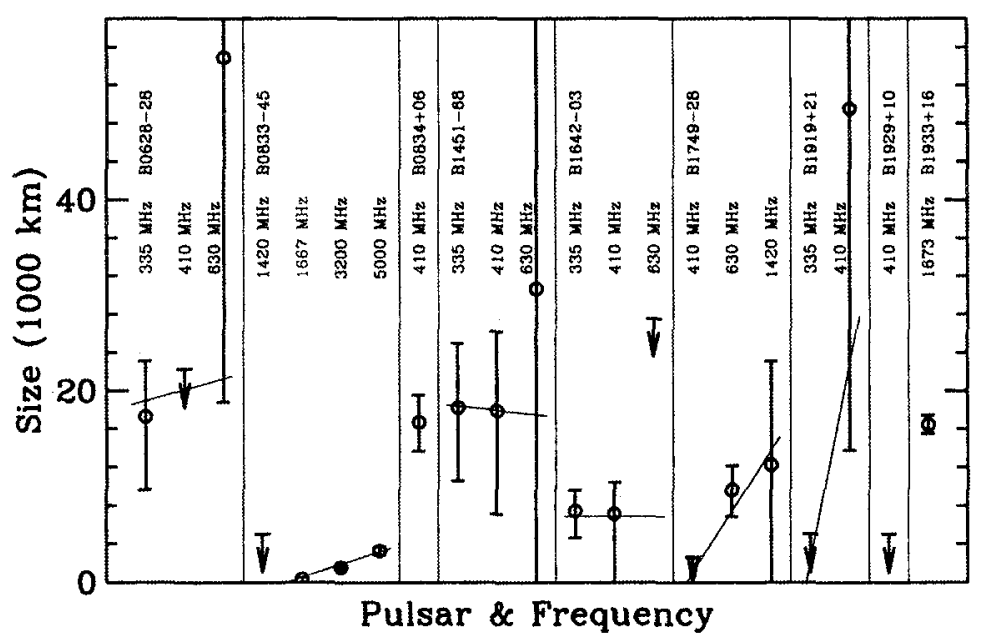

Figure 2. Sizes of pulsars inferred from the modulation indices measured by Roberts and Ables (1982). Lines serve to guide the eye in relating sizes of one pulsar measured at different frequencies.

They found position shifts of $<850 \mathrm{~km}$ and $<1100 \mathrm{~km}$, respectively, between the 2 gates. More recently, Smirnova et al. (1996) (compared the scintillation pattern in many gates across the pulse, for the strong pulsars B0834+06, B 1133+16, $\mathrm{B} 1237+25$, and $\mathrm{B} 1919+21$, at $f=103 \mathrm{MHz}$. They observed significant and, indeed, quite marked changes in the diffraction pattern between pulse phases, implying a shift in source position of $10^{3}$ to $10^{4} \mathrm{~km}$ over the pulse.

\subsection{SMEARING OF SCINTILLATIONS BY SOURCE SIZE}

For a point source in strong scattering, the modulation index is $m=\left(\left\langle l^{2}\right\rangle-\right.$ $\left.(\langle I\rangle)^{2}\right) /(\langle I\rangle)^{2}=1$, and the distribution of intensity is exponential. For an extended source, the modulation index is $<1$, and the distribution of intensity is more condensed (Gwinn et al., 1998). Fits to the distribution provide a more accurate measure of source size, and are less susceptible to systematic effects that can affect the distribution of intensity.

Roberts and Ables (1982) measured modulation indices for 9 pulsars, at $f=$ 410 to $3500 \mathrm{MHz}$. Figure 2 shows their results. They find source sizes of 0 to $4 \times 10^{4} \mathrm{~km}$. As the figure shows, they find little evidence for larger size at lower frequency. Gwinn et al. $(1997,2000)$ fit a model to the distribution of interferometric visibility, and found sizes for the Vela pulsar in 3 gates across the pulse. The size decreases with pulse phase, from $440 \pm 90 \mathrm{~km}$ to less than $200 \mathrm{~km}$, at $f=2300 \mathrm{MHz}$. Macquart et al. (2000) find a much smaller size for this pulsar at $f=660 \mathrm{MHz}$. Their innovative techniques allowed them to study the extremely narrow-bandwidth scintillation of the Vela pulsar at this frequency, Their observed 


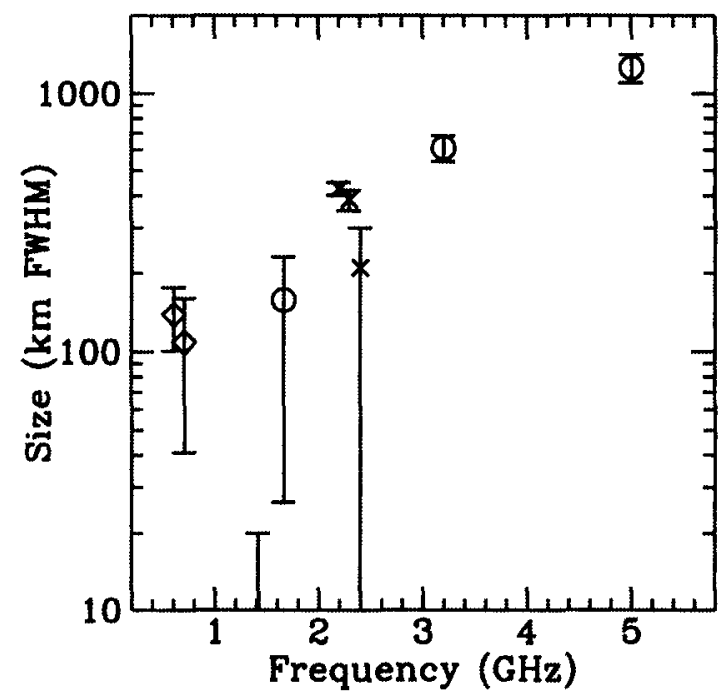

Figure 3. Size of the Vela pulsar as determined by various groups, plotted against observing frequency. Circles: Roberts and Ables (1982); crosses ( 3 pulse gates): Gwinn et al. (2000); diamonds (2 linear polarizations): Macquart et al. (2000).

modulation indices in the 2 senses of linear polarization correspond to inferred sizes of $140 \pm 40$ and $100 \pm 70 \mathrm{~km}$, using the screen location found by Desai et al. (1992). Interestingly, Roberts and Ables inferred increasing size for this pulsar as a function of observing frequency. Figure 3 summarizes size measurements for the Vela pulsar. The results suggest a nearly constant, or possibly increasing, size for the emission region as a function of observing frequency.

\section{Summary}

Three techniques yield sizes for pulsar emission regions of $10^{2}$ to $10^{4} \mathrm{~km}$. Comparison of the sizes inferred for individual pulsars at different frequencies show little evidence for a decrease in size with increasing observing frequency, contrary to expectation from the radius-to-frequency mapping. The observations are consistent with constant, or even increasing, size with observing frequency.

The primary limitations of present measurements are uncertainty of the distance of the scatterer, and signal-to-noise ratio. The distance of the scatterer relates the linear scales at the source and observer, analogously to the magnification of a lens. Interferometric and single-dish observations can help to determine this distance, reducing uncertainties for strong pulsars.

To obtain useful data, the signal-to-noise ratio must reach 1 in a single scintle (scintillation bandwidth $\times$ scintillation timescale), and an observation must contain 
a statistically significant number of scintles. Even with the largest telescopes, this is possible only for strong pulsars with convenient scattering parameters. Construction of telescopes significantly larger than any now operating will allow determination of sizes for a much larger group of pulsars.

\section{References}

Cordes, J.M., Weisberg, J.M. and Boriakoff, V.: 1983, Astrophys. J. 268, 370.

Desai, K.M., Gwinn, C.R., Reynolds, J.R., King, E.A., Jauncey, D., Flanagan, C., Nicolson, G., Preston, R.A. and Jones, D.L.: 1992, Astrophys. J. 393, L75.

Gupta, Y., Bhat, N.D.R. and Rao, A.P.: 1999, Astrophys. J. 520, 173.

Gwinn, C.R., Britton, M.C., Reynolds, J.R., Jauncey, D.L., King, E.A., McCulloch, P.M., Lovell, J.E.J., Flanagan, C.S. and Preston, R.A.: 1998, Astrophys. J. 505, 928.

Gwinn, C.R., Britton, M.C., Reynolds, J.E., Jauncey, D.L., King, E.A., McCulloch, P.M., Lovell, J.E.J., Flanagan, C.S. and Preston, R.A.: 2000, Astrophys. J. 531, 902.

Gwinn, C.R., Ojeda, M.J., Britton, M.C., Reynolds, J.R., Jauncey, D.L., King, E.A., McCulloch, P.M., Lovell, J.E.J., Flanagan, C.S., Smits, D.P., Preston, R.A. and Jones, D.L.: 1997, Astrophys. J. 483, 53.

Macquart, J.-P., Johnston, S., Walker, M. and Stinebring, D.: 2000, in: M. Kramer, N. Wex and N. Wielebinski (eds.), Pulsar Astronomy - 2000 and Beyond, Astronomical Society of the Pacific, p. 215.

Phillips, J.A.: 1992, Astrophys. J. 385, 282.

Roberts, J.A. and Ables, J.G.: 1982, Mon. Not. R. Astron. Soc. 201, 1119.

Smirnova, T.V., Shishov, V.I., and Malofeev, V.M.: 1996, Astrophys. J. 462, 289.

Wolszczan, A., Bartlett, J.E. and Cordes, J.M.: 1988, in: J.M. Cordes, B.J. Rickett and D.C. Backer (eds.), Radio Wave Scattering in the Interstellar Medium, American Institute of Physics, p. 145.

Wolszczan, A. and Cordes, J.M.: 1987, Astrophys. J. 320, L35. 